\title{
Wireless Channel Allocation Using an Auction Algorithm
}

\author{
Jun Sun, Eytan Modiano, Senior Member, IEEE, and Lizhong Zheng, Member, IEEE
}

\begin{abstract}
We develop a novel auction-based algorithm to allow users to fairly compete for a wireless fading channel. We use the second-price auction mechanism whereby user bids for the channel, during each time slot, based on the fade state of the channel, and the user that makes the highest bid wins use of the channel by paying the second highest bid. Under the assumption that each user has a limited budget for bidding, we show the existence of a Nash equilibrium strategy, and the Nash equilibrium leads to a unique allocation for certain channel state distribution, such as the exponential distribution and the uniform distribution over $[0,1]$. For uniformly distributed channel state, we establish that the aggregate throughput received by the users using the Nash equilibrium strategy is at least $3 / 4$ of what can be obtained using an optimal centralized allocation that does not take fairness into account. We also show that the Nash equilibrium strategy leads to an allocation that is Pareto optimal (i.e., it is impossible to make some users better off without making some other users worse off). Based on the Nash equilibrium strategies of the second-price auction with money constraint, we further propose a centralized opportunistic scheduler that does not suffer the shortcomings associated with the proportional fair scheduler.
\end{abstract}

Index Terms-Auction, channel allocation, wireless communication.

\section{INTRODUCTION}

$\mathbf{N}$ ETWORK resources such as bandwidth and power are usually limited in wireless and satellite networks. A systematic procedure in place for fair resource allocation, therefore, is often desirable. However, there is no consensus on the notion of fairness. Any centrally imposed notion of fairness may be against an individual user's interest. In this paper, we address the problem of fair resource allocation by allowing individual users to compete for resources through bidding for the use of the channel.

A fundamental characteristic of a wireless network is that the channel over which communication takes place is often timevarying. This variation of the channel quality is due to constructive and destructive interference between multipaths and shadowing effects (fading). In a single cell with one transmitter (base station or satellite) and multiple users communicating through fading channels, the transmitter can send data at higher rates to users with better channels. In a time-slotted system, time slots are allocated among users according to their channel qualities.

Manuscript received February 15, 2005; revised November 15, 2005. This work was supported in part by the NASA Space Communication Project under Grant NAG3-2835.

The authors are with the Laboratory for Information and Decision Systems, Massachusetts Institute of Technology, Cambridge, MA 02139-4307 USA (e-mail: junsun@mit.edu; modiano@mit.edu; lizhong@mit.edu).

Digital Object Identifier 10.1109/JSAC.2006.872890
TABLE I

ThroughPUT RESUlTS USING DIFFERENT NOTIONS OF FAIRNESS

\begin{tabular}{|l||c|c|c|}
\hline & $\begin{array}{c}\text { Throughput } \\
\text { for A }\end{array}$ & $\begin{array}{c}\text { Throughput } \\
\text { for B }\end{array}$ & $\begin{array}{c}\text { Total } \\
\text { throughput }\end{array}$ \\
\hline \hline No fair constraint & 0 & 1.2 & 1.2 \\
\hline Maxmin fair & 0.2 & 0.3 & 0.5 \\
\hline Time fraction & 0.1 & 0.9 & 1.0 \\
\hline
\end{tabular}

The problem of resource allocation in wireless networks has received much attention in recent years. In [1], the authors try to maximize the data throughput of an energy and time constrained transmitter communicating over a fading channel. A dynamic programming formulation that leads to an optimal transmission schedule is presented. Other works address the similar problem, without consideration of fairness, include [7] and [8]. In [5], the authors consider scheduling policies for maxmin fairness allocation of bandwidth, which maximizes the allocation for the most poorly treated sessions, while not wasting any network resources, in wireless ad hoc networks. In [4], the authors designed a scheduling algorithm that achieves proportional fairness, a notion of fairness originally proposed by Kelly [6]. In [9], the authors present a slot allocation that maximizes expected system performance subject to the constraint that each user gets a fixed fraction of time slots. The authors did not use a formal notion of fairness, but argue that their system can explicitly set the fraction of time assigned to each user. Hence, while each user may get to use the channel an equal fraction of the time, the resulting throughput obtained by each user may be vastly different.

The following simple example illustrates the different allocations that may result from the different notions of fairness. We consider the communication system with one transmitter and two users, A and B, and the allocations that use different notions of fairness discussed in the previous paragraph. We assume that the throughput is proportional to the channel condition. The channel coefficient, which is a quantitative measure of the channel condition ranging from 0 to 1 with 1 as the best channel condition, for user A and user B in the two time slots are $(0.1,0.2)$ and $(0.3,0.9)$, respectively. The throughput result for each individual user and for total system under different notions of fairness constraint are given in Table I. When there is no fairness constraint, to maximize the total system throughput would require the transmitter to allocate both time slots to user B. To achieve maxmin fair allocation, the transmitter would allocate slot one to user B and slot two to user A, thus resulting in a total throughput of 0.5 . If the transmitter wants to maximize the total throughput subject to the constraint that each user gets one time slot (i.e., the approach of [9]), the resulting allocation, denoted as time fraction fair, is to give user A slot one and user B slot two. 
As a result, the total throughput is 1.0. In the above example, the transmitter selects an allocation to ensure an artificially chosen notion of fairness. From Table I, we can see that from the user's perspective, no notion is truly fair as both users want slot two. In order to resolve this conflict, we use a new approach which allows users to compete for time slots. In this way, each user is responsible for its own action and its resulting throughput. We call the fraction of bandwidth received by each user competitive fair. Using this notion of competitive fairness, the resulting throughput obtained for each user can serve as a reference point for comparing various other allocations. Moreover, the competitive fair allocation scheme can provide fundamental insight into the design of a fair scheduler that make sense.

In our model, users compete for time slots. For each time slot, each user has a different valuation (i.e., its own channel condition). And each user is only interested in getting a higher throughput for itself. Naturally, these characteristics give rise to an auction. In this paper, we consider the second-price auction mechanism. Using the second-price auction mechanism, users submit a "bid" for the time slot and the transmitter allocates the slot to the user that made the highest bid. Moreover, in the second-price auction mechanism, the winner only pays the second highest bid [13]. The second-price auction mechanism is used here due to its "truth telling" nature (i.e., it is optimal for a user to bid its true valuation of a particular object). Each user is assumed to have an initial amount of money. The money possessed by each user can be viewed as fictitious money that serves as a mechanism to differentiate the quality-of-service (QoS) given to the various users. This fictitious money, in fact, could correspond to a certain QoS for which the user paid in real money. As for the solution of the slot auction game, we use the concept of Nash equilibrium, which is a set of strategies (one for each player) from which there are no profitable unilateral deviation.

In this paper, we consider a communication system with one transmitter and multiple users. For each time slot, channel states are independent and identically distributed with known probability distribution. Each user wants to maximize its own $e x$ pected throughput subject to an average money constraint. Our major results include the following.

- We find the Nash equilibrium strategy for general channel state distribution.

- We show that the Nash equilibrium strategy pair leads to a unique allocation for certain channel state distribution, such as the exponential distribution and the uniform distribution over $[0,1]$.

- We show that the Nash equilibrium strategy of this auction leads to an allocations at which total throughput is no worse than $3 / 4$ of the throughput obtained by an algorithm that attempts to maximize total system throughput without a fairness constraint under uniform distribution.

- We show that the Nash equilibrium strategy leads to an allocation that is Pareto optimal (i.e., it is impossible to make some users better off without making some other users worse off).

- Based on the Nash equilibrium strategies of the secondprice auction with money constraint, we also propose a centralized opportunistic scheduler that does not suffer the shortcomings associated with the proportional fair and the time fraction fair scheduler.
Game theoretical approaches to resource allocation problems have been explored by many researchers recently (e.g., [2] and [12]). In [2], the authors consider a resource allocation problem for a wireless channel, without fading, where users have different utility values for the channel. They show the existence of an equilibrium pricing scheme where the transmitter attempts to maximize its revenue and the users attempt to maximize their individual utilities. In [12], the authors explore the properties of a congestion game where users of a congested resource anticipate the effect of their action on the price of the resource. Again, the work of [12] focuses on a wireline channel without the notion of wireless fading. Our work attempts to apply game theory to the allocation of a wireless fading channel. In particular, we show that auction algorithms are well suited for achieving fair allocation in this environment. Other papers dealing with the application of game theory to resource allocation problems include [3], [16], and [17].

This paper is organized as follows. In Section II, we describe the communication system and the auction mechanism. In Section III, we start by presenting the Nash equilibrium strategy pair for the two users game with general channel distribution. The uniqueness of the allocation scheme derived from the Nash equilibrium is shown when the channel state has the exponential or the uniform $[0,1]$ distribution. We then derive the Nash equilibrium for the $N$-users game. In Section IV, we show the unique Nash equilibrium strategy for the case that each user can use multiple bidding functions. The Pareto optimality of the allocation resulting from the Nash equilibrium strategies is established in Section V. In Section VI, we compare the throughput results of the Nash equilibrium strategy with other centralized allocation algorithms. Finally, Section VII concludes the paper.

\section{PROBlem Formulation}

We consider a communication scenario where a single transmitter sends data to $N$ users over independent fading channels. We assume that there is always data to be sent to the users. Time is assumed to be discrete, and the channel state for a given channel changes according to a known probabilistic model independently over time. The transmitter can serve only one user during a particular slot with a constant power $P$. The channel fade state thus determines the throughput that can be obtained.

For a given power level, we assume for simplicity that the throughput is a linear function of the channel state. This can be justified by the Shannon capacity at low signal-to-noise ratio [1]. However, for general throughput function, it can be shown that the method used in this paper applies as well. Let $X_{i}$ be a random variable denote the channel state for the channel between the transmitter and user $i, i=1, \cdots, N$. When transmitting to user $i$, the throughput will then be $P \cdot X_{i}$. Without loss of generality, we assume $P=1$ throughout this paper.

We now describe the second-price auction rule used in this paper. Let $\alpha_{i}$ be the average amount of money available to user $i$ during each time slot. We assume that the values of $\alpha_{i}$ 's are known to all users. Moreover, users know the distribution of $X_{i}$ for all $i$. We also assume that the exact value of the channel state $X_{i}$ is revealed to user $i$ only at the beginning of each time slot. During each time slot, the following actions take 
place: 1) each user submits a bid according to the channel condition revealed to it; 2) the transmitter chooses the one with the highest bid to transmit; and 3) the price that the winning user pays is the second highest bidder's bid. Users who lose the bid do not pay. In case of a tie, the winner is chosen among the equal bidders with equal probability. Formally, this $N$-players game can be written as $\Gamma=\left[N,\left\{S_{i}\right\},\left\{g_{i}(\cdot)\right\}\right]$ which specifies for each player $i$ a set of strategies, or bidding functions, $S_{i}$ (with $s_{i} \in S_{i}$ ) and a payoff function $g_{i}\left(s_{1}, \cdots, s_{N}\right)$ giving the throughput associated with outcome of the auction arising from strategies $\left(s_{1}, \cdots, s_{N}\right)$.

The formulation of our auction is different from the type of auction used in economic theory in several ways. First, we look at a case where the number of object (time slots) in the auction goes to infinity (average cost criteria). While in the current auction research, the number of object is finite [13]-[15]. Second, in our auction formulation, the money used for bidding does not have a direct connection with the value of the time slot. Money is merely a tool for users to compete for time slots, and it has no value after the auction. Therefore, it is desirable for each user to spend all of its money. However, in the traditional auction theory, an object's value is measured in the same unit as the money used in the bidding process, hence their objective is to maximize the difference between the object's value and its cost.

Besides the second-price auction, the first-price auction and the all-pay auction are two other commonly used auction mechanisms. In the first-price auction, each bidder submits a single bid without seeing the others' bids, and the object is sold to the bidder who makes the highest bid. Only the winner pays its bid. In the all-pay auction, each user independently submits a single bid without seeing the others' bids, and the object is given to the bidder who makes the highest bid. However, all users pay their bid regardless of whether they win or loss [13]. We choose to use the second-price auction in this paper to illustrate the auction approach to resource allocation in wireless networks. As we will see later, second-price auction results in an allocation that is efficient. More specifically, it is Pareto optimal.

The objective for each user is to design a bidding strategy, which specifies how a user will act in every possible distinguishable circumstance, to maximize its own expected throughput per time slot subject to the expected or average money constraint. Once a user, say user 1 , chooses a function, say $f_{1}$, to be its strategy, it bids an amount of money equal to $f_{1}(x)$ when it sees its channel condition is $X_{1}=x$.

\section{NASh EQUilibrium Under SECOND-PRICE AUCTION}

We begin our analysis of the second-price auction with an average money constraint by looking at a two-users case for simplicity. Specifically, we present here a Nash equilibrium strategy pair $\left(f_{1}^{*}, f_{2}^{*}\right)$ for the second-price auction under general channel distribution. We consider here the case where users choose their strategies from the set $F_{1}$ and $F_{2}$, respectively. Each user's strategy is a function of its own channel state $X_{i}$. Thus, $F_{i}$ is defined to be the set of continuous real-valued, and square integrable functions over the support of $X_{i}$. Without loss of generality, we further assume functions in $F_{i}$ to be increasing. We define $A:\left(x_{1}, x_{2}\right) \rightarrow\{1,2\}$ to be an allocation that maps the possible channel state realization, $\left(x_{1}, x_{2}\right)$, to either user 1 or user 2 . Here, we are interested in the allocation that result from the Nash equilibrium strategies.

We first consider a channel state $X_{i}$ that is continuously distributed over a finite interval $\left[l_{i}, u_{i}\right]$ where $l_{i}$ and $u_{i}$ are nonnegative real number with $u_{i}>l_{i}$. Later, we will consider the case that $u_{i}$ is infinite (e.g., when $X_{i}$ is exponentially distributed).

To find the Nash equilibrium strategy pair, we use the following approach. Given user 1's strategy $f_{1} \in F_{1}$ with its range from $f_{1}\left(l_{1}\right)=a$ to $f_{1}\left(u_{1}\right)=b$, user 2 wants to maximize its own expected throughput, while satisfying its expected money constraint. For a given $f_{1}$, if user 2 chooses a bidding function $f_{2}$, the expected throughput or payoff function for user 2 is given by

$$
g_{2}\left(f_{1}, f_{2}\right)=E_{X_{1}, X_{2}}\left[X_{2} \cdot 1_{f_{2}\left(X_{2}\right) \geq f_{1}\left(X_{1}\right)}\right]
$$

where

$$
1_{f_{2}\left(X_{2}\right) \geq f_{1}\left(X_{1}\right)}=\left\{\begin{array}{ll}
1, & \text { if } f_{2}\left(X_{2}\right) \geq f_{1}\left(X_{1}\right) \\
0, & \text { otherwise }
\end{array} .\right.
$$

Recall that in the second-price auction rule, the price that the winner pays is actually the second highest bid. Therefore, the set of feasible bidding functions for user 2, denoted as $S_{2}\left(f_{1}\right)$, is given by

$$
S_{2}\left(f_{1}\right)=\left\{f_{2} \in F_{2} \mid E_{X_{1}, X_{2}}\left[f_{1}\left(X_{1}\right) \cdot 1_{f_{2}\left(X_{2}\right) \geq f_{1}\left(X_{1}\right)}\right] \leq \alpha_{2}\right\} .
$$

Note that the inverse function $f_{1}^{-1}(y)$ may not be well defined for $y \in[a, b]$ since $f_{1}$ may not be strictly increasing over $[a, b]$. Therefore, to avoid such problem, we define the following function:

$$
h(y)= \begin{cases}l_{1}, & \text { if } y \leq a \\ \max \left\{x \mid f_{1}(x) \leq y\right\}, & \text { if } a<y<b . \\ u_{1}, & \text { if } y \geq b\end{cases}
$$

In the special case that $f_{1}$ is strictly increasing, $h(y)$ is reduced to the following:

$$
h(y)= \begin{cases}l_{1}, & \text { if } y \leq a \\ f_{1}^{-1}(y), & \text { if } a<y<b . \\ u_{1}, & \text { if } y \geq b\end{cases}
$$

For the rest of thise paper, it is convenient to consider the definition of $h(y)$ given in (4).

We say the strategy $f_{2}$ is a best response for player 2 to his rival's strategy $f_{1}$ if $g_{2}\left(f_{1}, f_{2}\right) \geq g_{2}\left(f_{1}, f_{2}^{\prime}\right)$ for all $f_{2}^{\prime} \in S_{2}\left(f_{1}\right)$. A strategy pair $\left(f_{1}^{*}, f_{2}^{*}\right)$ is said to be in Nash equilibrium if $f_{1}^{*}$ is the best response for user 1 to user 2's strategy $f_{2}^{*}$, and $f_{2}^{*}$ is the best response for user 2 to user 1's strategy $f_{1}^{*}$. The following theorem characterizes the best response of user 2 to a fixed user 1 's strategy.

Theorem 1: Given user 1's bidding strategy $f_{1} \in F_{1}$ with its range from $f_{1}\left(l_{1}\right)=a$ to $f_{1}\left(u_{1}\right)=b$, user 2 's best response has the following form:

$$
\begin{array}{lll}
f_{2}\left(x_{2}\right) \leq a, & \text { for } & x_{2} \in\left[l_{2}, \theta_{1}\right] \\
f_{2}\left(x_{2}\right)=c_{2} \cdot x_{2}, & \text { for } & x_{2} \in\left[\theta_{1}, \theta_{2}\right] \\
f_{2}\left(x_{2}\right) \geq b, & \text { for } & x_{2} \in\left[\theta_{2}, u_{2}\right]
\end{array}
$$

where $\theta_{1}, \theta_{2} \in\left[l_{2}, u_{2}\right]$ and $c_{2} \cdot \theta_{1}=a, c_{2} \cdot \theta_{2}=b$. 
Proof: Given user 1's bidding strategy $f_{1}$ and user 2's bid at a particular time slot is $y$, the probability that user 2 wins this slot, denoted as $P_{2}^{\text {win }}(y)$, is given by

$$
\begin{aligned}
P_{2}^{\text {win }}(y) & =P\left(f_{1}\left(X_{1}\right) \leq y\right)=P\left(X_{1} \leq h(y)\right) \\
& =\int_{l_{1}}^{h(y)} p_{X_{1}}\left(x_{1}\right) d x_{1} .
\end{aligned}
$$

Therefore, the optimization problem that user 2 faces is to find a strategy $f_{2}$ that maximize its expected throughput, which can be written as the following:

$$
\begin{aligned}
& \max _{f_{2}} \int_{l_{2}}^{u_{2}} x_{2} p_{X_{2}}\left(x_{2}\right) P_{2}^{\text {win }}\left(f_{2}\left(x_{2}\right)\right) d x_{2} \\
& \quad=\max _{f_{2}} \int_{l_{2}}^{u_{2}} x_{2} p_{X_{2}}\left(x_{2}\right) \int_{l_{1}}^{h\left(f_{2}\left(x_{2}\right)\right)} p_{X_{1}}\left(x_{1}\right) d x_{1} d x_{2} \\
& \text { subj. to } \int_{l_{2}}^{u_{2}} \int_{l_{1}}^{h\left(f_{2}\left(x_{2}\right)\right)} f_{1}\left(x_{1}\right) p_{X_{1}}\left(x_{1}\right) p_{X_{2}}\left(x_{2}\right) d x_{1} d x_{2} \\
& \quad \leq \alpha_{2}
\end{aligned}
$$

where the integration is over the region that user 2's bid is higher than user 1's bid. The constraint term denotes the expected money that user 2 has to pay over the region which it has a higher bid than user 1 . To solve the above optimization problem, we use the optimality condition in [11]. First, we write the Lagrangian function below

$$
\begin{aligned}
& \int_{l_{2}}^{u_{2}} \int_{l_{1}}^{h\left(f_{2}\left(x_{2}\right)\right)} x_{2} p_{X_{1}}\left(x_{1}\right) p_{X_{2}}\left(x_{2}\right) d x_{1} d x_{2}- \\
& \lambda_{2}\left(\int_{l_{2}}^{u_{2}} \int_{l_{1}}^{h\left(f_{2}\left(x_{2}\right)\right)} f_{1}\left(x_{1}\right) p_{X_{1}}\left(x_{1}\right) p_{X_{2}}\left(x_{2}\right) d x_{1} d x_{2}-\alpha_{2}\right) \\
& =\int_{l_{2}}^{u_{2}}\left[\int_{l_{1}}^{h\left(f_{2}\left(x_{2}\right)\right)}\left(x_{2}-\lambda_{2} f_{1}\left(x_{1}\right)\right) p_{X_{1}}\left(x_{1}\right) d x_{1}\right] p_{X_{2}}\left(x_{2}\right) d x_{2}
\end{aligned}
$$$$
-\lambda_{2} \alpha_{2} \text {. }
$$

We then choose a function $f_{2}$ to maximize the above equation. Also, a positive $\lambda_{2}$, which depends on $f_{1}$, is chosen such that the inequality constraint is met with equality. Specifically, for each value $x_{2}$, we solve for the optimal $f_{2}\left(x_{2}\right)$

$$
\max _{f_{2}\left(x_{2}\right)} \int_{l_{1}}^{h\left(f_{2}\left(x_{2}\right)\right)}\left(x_{2}-\lambda_{2} f_{1}\left(x_{1}\right)\right) p_{X_{1}}\left(x_{1}\right) d x_{1} .
$$

For convenience, we let $z=f_{2}\left(x_{2}\right)$. Then, (8) becomes

$$
\max _{z} L_{1}(z)=\int_{l_{1}}^{h(z)}\left(x_{2}-\lambda_{2} f_{1}\left(x_{1}\right)\right) p_{X_{1}}\left(x_{1}\right) d x_{1} .
$$

For a fixed $x_{2}$, the term $x_{2}-\lambda_{2} f_{1}\left(x_{1}\right)$ is a decreasing function in $x_{1}$ since $f_{1}\left(x_{1}\right)$ is increasing. To maximize $L_{1}(z)$, it is equivalent to choosing a value for $h\left(z^{*}\right)$ that includes all value of $x_{1}$ such that $x_{2}-\lambda_{2} f_{1}\left(x_{1}\right)$ is positive, or maximizes the area under the curve $x_{2}-\lambda_{2} f_{1}\left(x_{1}\right)$. It is apparent that the optimal value $z^{*}$ should be chosen such that $x_{2}-\lambda_{2} f_{1}\left(h\left(z^{*}\right)\right)=0$ or $z^{*}=x_{2} / \lambda_{2}$. However, if $x_{2}-\lambda_{2} f_{1}(h(z))>0$ for all $z \in[a, b]$, we let $z^{*} \geq b$. Similarly, if $x_{2}-\lambda_{2} f_{1}(h(z))<0$ for all $z \in[a, b]$, we let $z^{*} \leq a$. Thus, from (3), we see that the optimal bidding function has the following form:

$$
\begin{array}{lll}
f_{2}\left(x_{2}\right) \leq a, & \text { for } & x_{2} \in\left[l_{2}, \theta_{1}\right] \\
f_{2}\left(x_{2}\right)=c_{2} \cdot x_{2}, & \text { for } & x_{2} \in\left[\theta_{1}, \theta_{2}\right] \\
f_{2}\left(x_{2}\right) \geq b, & \text { for } & x_{2} \in\left[\theta_{2}, u_{2}\right]
\end{array}
$$

where $\theta_{1}, \theta_{2} \in\left[l_{2}, u_{2}\right]$ and $c_{2} \cdot \theta_{1}=a, c_{2} \cdot \theta_{2}=b$.
The above theorem indicates that for user 2 to maximize its throughput given user 1's strategy $f_{1}$, the optimal strategy may not be unique following the definition of the Nash equilibrium. For $x_{2} \in\left[l_{2}, \theta_{1}\right]$, as long as $f_{2}\left(x_{2}\right) \leq a$, user 2 always loses the bid, and the throughput for user 2 does not change. However, from second-price bidding rule, user 2's strategy affects user 1's strategy through the expected budget constraint that user 1 must satisfy. This way, user 2 will choose $f_{2}\left(x_{2}\right)=a$ for $x_{2} \in\left[l_{2}, \theta_{1}\right]$. Intuitively, even if user 2 knows that it will not win a particular time slot, it will still choose to maximize its bid in order to force user 1 to pay more. Hence, user 2's best response is in this sense unique. Therefore, although the second-price auction with average money constraint does not in general have an unique Nash equilibrium, it does have an unique outcome. We will elaborate on this more in Section III-B where the users' channel distributions are different.

Similarly, given user 2's bidding function $f_{2}$, we can carry out the same analysis to find that the best response for user 1 has the form $f_{1}\left(x_{1}\right)=c_{1} \cdot x_{1}$. The next theorem shows that indeed we can always find a pair $c_{1}$ and $c_{2}$ such that both users' money constraints are satisfied simultaneously, and thus show the existence of a Nash equilibrium strategy pair.

Theorem 2: A Nash equilibrium exists in the second-price auction game $\Gamma=\left[2,\left\{S_{i}\right\},\left\{g_{i}(\cdot)\right\}\right]$ with $\left\{S_{i}\right\}$ and $\left\{g_{i}(\cdot)\right\}$ defined in (2) and (1), respectively.

Proof: For the channel state $X_{1}$ distributed over the interval $\left[l_{1}, u_{1}\right]$, the best response given in (5) indicates that $f_{1}\left(x_{1}\right)=c_{1} \cdot x_{1}$ for all $x_{1}$ in $\left[l_{1}, u_{1}\right]$ is a valid best response.

Without loss of generality, we consider only linear bidding functions (i.e., $f_{1}\left(x_{1}\right)=c_{1} \cdot x_{1} \forall x_{1} \in\left[l_{1}, u_{1}\right], f_{2}\left(x_{2}\right)=$ $c_{2} \cdot x_{2} \forall x_{2} \in\left[l_{2}, u_{2}\right]$ and $\left.c_{1}, c_{2} \in[0, \infty)\right)$ for the purpose of showing the existence of a Nash equilibrium strategy pair. A Nash equilibrium exists if we can find a pair of $c_{1}$ and $c_{2}$ which satisfy the following two constraints:

$$
\begin{aligned}
& E_{X_{1}, X_{2}}\left[f_{2}\left(X_{2}\right) \cdot 1_{f_{1}\left(X_{1}\right) \geq f_{2}\left(X_{2}\right)}\right] \leq \alpha_{1} \\
& E_{X_{1}, X_{2}}\left[f_{1}\left(X_{1}\right) \cdot 1_{f_{2}\left(X_{2}\right) \geq f_{1}\left(X_{1}\right)}\right] \leq \alpha_{2} .
\end{aligned}
$$

Given user 2's strategy $f_{2}\left(x_{2}\right)=c_{2} \cdot x_{2}$, we define the set $S_{1}\left(c_{2}\right)$ to be the set of feasible strategy for user 1 . Specifically, $S_{1}\left(c_{2}\right)=\left\{c_{1} \in[0, \infty) \mid E_{X_{1}, X_{2}}\left[c_{2} X_{2} \cdot 1_{c_{1} X_{1} \geq c_{2} X_{2}}\right] \leq \alpha_{1}\right\}$. The best response for user 1 when user 2 chooses $c_{2}, b_{1}\left(c_{2}\right)$, is given by

$$
b_{1}\left(c_{2}\right)=\arg \max _{y \in S_{1}\left(c_{2}\right)} E_{X_{1}, X_{2}}\left[X_{1} \cdot 1_{y X_{1} \geq c_{2} X_{2}}\right] .
$$

To show that Nash equilibrium exists, we need to show that the best response correspondence $b_{1}(\cdot)$ is nonempty, convex-valued, and upper hemicontinuous [19]. Note first that $b_{1}\left(c_{2}\right)$ is the set of maximizer of a continuous function, here the function $E_{X_{1}, X_{2}}\left[X_{1} \cdot 1_{y X_{1} \geq c_{2} X_{2}}\right]$, on a compact set $S_{1}\left(c_{2}\right)$. Hence, it is nonempty. The convexity of $b_{1}\left(c_{2}\right)$ follow because the set of maximizers of a quasi-concave function, i.e., $E_{X_{1}, X_{2}}\left[X_{1} \cdot 1_{y X_{1} \geq c_{2} X_{2}}\right]$, on a convex set (here $S_{1}\left(c_{2}\right)$ ) is convex. $E_{X_{1}, X_{2}}\left[X_{1} \cdot 1_{y X_{1} \geq c_{2} X_{2}}\right]$ is quasi-concave because it is nondecreasing in $y$. Finally, since the set $S_{1}\left(c_{2}\right)$ is compact for all $c_{2} \in[0, \infty)$, following the Berge Maximum Theorem [20], we have $b_{1}\left(c_{2}\right)$ is upper hemicontinuous. Now, all the conditions of the Kalkutani fixed point theorem are satisfied [19]. Hence, there exists a Nash equilibrium for this game. 
The Nash equilibrium strategy discussed above is in general not unique. However, under a continuous channel state distribution that starts with zero, such as the uniform distribution over $[0,1]$ or the exponential distribution, the Nash equilibrium bidding strategies are unique and lead to an unique allocation. Next, we will discuss the Nash equilibrium strategy pair of these two distribution.

\section{A. Uniform Channel Distribution}

In this section, we examine the two users game with the channel state $X_{i}$ uniformly distributed over [0,1]. Following the approach discussed in the previous section, we find the unique allocation resulting from the Nash equilibrium strategy. Given a strategy pair $\left(f_{1}^{*}, f_{2}^{*}\right)$ to be in Nash equilibrium, we first investigate the bids that users submit when the channel state $X_{i}$ is equal to 0 (i.e., the value of $f_{1}^{*}(0)$ and $f_{2}^{*}(0)$ ). The result is stated in the following lemma.

Lemma 1: For a strategy pair $\left(f_{1}^{*}, f_{2}^{*}\right)$ to be a Nash equilibrium strategy pair, we must have $f_{1}^{*}(0)=f_{2}^{*}(0)=0$ when the channels are uniformly distributed over $[0,1]$.

Proof: We consider the following three cases regarding the bidding strategy when the channel state is at 0 .

- Case 1) $f_{1}^{*}(0)=0$ and $f_{2}^{*}(0)=0$.

- Case 2) $f_{1}^{*}(0)=a$ with $a>0$ and $f_{2}^{*}(0)=0$, or $f_{1}^{*}(0)=$ 0 and $f_{2}^{*}(0)=a$ with $a>0$.

- Case 3) $f_{1}^{*}(0)=a$ and $f_{2}^{*}(0)=b$ with $a>0$ and $b>0$.

Case 2 cannot be true from the discussion in the previous section. To see this, consider $f_{1}^{*}(0)=a$ with $a>0$ and $f_{2}^{*}(0)=0$. Given a time slot with user 1's channel states $x_{1}=0$, the expected money user 1 has to pay is positive since user 2's bidding function is continuous and $f_{2}^{*}(0)=0$. But the expected throughput rewarded for that time slot is zero for user 1 . Hence, user 1 should bid zero when its channel state is zero. Similar idea can be used to show that case 3 is also impossible. Given a time slot with user 1's channel state $x_{1}=0$ and $f_{1}^{*}(0)=a \geq b$, the expected money user 1 has to pay is positive since user 2 's bidding function is continuous and $f_{2}^{*}(0)=b$. However, the expected throughput for that time slot is zero for user 1 . So user 1 would rather bid zero in this time slot.

With the above lemma, we can get the exact form of the Nash equilibrium strategy pair.

Theorem 3: With the channel states, $X_{1}$ and $X_{2}$, uniformly and independently distributed over $[0,1]$, the unique Nash equilibrium pair $\left(f_{1}^{*}, f_{2}^{*}\right)$ has the following form: $f_{1}^{*}\left(x_{1}\right)=c_{1} \cdot x_{1}$ and $f_{2}^{*}\left(x_{2}\right)=c_{2} \cdot x_{2}$, where $c_{1}$ and $c_{2}$ are chosen such that the expected money constraints are satisfied.

Proof: Combine Lemma 1 and the linear form of the bidding function shown previously, we have the above theorem.

We now calculate the exact value of $c_{1}$ and $c_{2}$. Without loss of generality, we assume that user 2 has more money than user 1 (i.e., $\alpha_{1}<\alpha_{2}$ ). Since the form of the optimal bidding strategy for both users is known, we need to get the exact value of $c_{1}$ and $c_{2}$ from the money constraint that users must satisfy. Thus, from (10) and (11), the constraint for user 1 is given by

$$
\begin{aligned}
\int_{0}^{1} \int_{0}^{f_{2}^{-1}\left(f_{1}\left(x_{1}\right)\right)} f_{2}\left(x_{2}\right) d x_{2} d x_{1}=\alpha_{1} \\
\Rightarrow \int_{0}^{1} \int_{0}^{\frac{c_{1}}{c_{2}} x_{1}} c_{2} \cdot x_{2} d x_{2} d x_{1}=\alpha_{1} .
\end{aligned}
$$

Note that the function $f_{1}^{-1}\left(f_{2}\left(x_{2}\right)\right)$ is well defined for $f_{2}\left(x_{2}\right) \in$ $\left[0, c_{1}\right]$. Therefore, the constraint for user 1 is given by:

$$
\begin{array}{r}
\int_{0}^{\frac{c_{1}}{c_{2}}} \int_{0}^{f_{1}^{-1}\left(f_{2}\left(x_{2}\right)\right)} f_{1}\left(x_{1}\right) d x_{1} d x_{2}+\int_{\frac{c_{1}}{c_{2}}}^{1} \int_{0}^{1} f_{1}\left(x_{1}\right) d x_{1} d x_{2}=\alpha_{2} \\
\int_{0}^{\frac{c_{1}}{c_{2}}} \int_{0}^{\frac{c_{2}}{c_{1} x_{2}}} c_{1} \cdot x_{1} d x_{1} d x_{2}+\int_{\frac{c_{1}}{c_{2}}}^{1} \int_{0}^{1} c_{1} \cdot x_{1} d x_{1} d x_{2}=\alpha_{2} .
\end{array}
$$

Solving the two equations, we get

$$
\begin{aligned}
& c_{1}=2\left(2 \alpha_{1}+\alpha_{2}\right) \\
& c_{2}=\frac{2\left(2 \alpha_{1}+\alpha_{2}\right)^{2}}{3 \alpha_{1}} .
\end{aligned}
$$

The throughput of each user is then given by

$$
\begin{aligned}
G_{1} & =\frac{\alpha_{1}}{\alpha_{2}+2 \alpha_{1}} \\
G_{2} & =\frac{1}{2}-\frac{3 \alpha_{1}^{2}}{2\left(\alpha_{2}+2 \alpha_{1}\right)^{2}} .
\end{aligned}
$$

Note that the linear bidding function leads to the following allocation: Given that the channel states are $x_{1}$ and $x_{2}$ during a time slot, the transmitter assigns the slot to user 1 if $x_{1} \geq c \cdot x_{2}$, where $c=c_{2} / c_{1}$, and to user 2, otherwise. We will see later that this form of allocation leads to the Pareto optimality.

\section{B. The Unique Outcome of the Game}

As we mentioned previously, the Nash equilibrium is not unique in general (although unique for the cases where channel states are exponentially distributed or uniformly distributed over $[0,1])$; however, the outcome of this second-price auction with money constraint is unique. To see this, consider an example where $X_{1}$ is uniformly distributed over the interval $[0,10]$, and $X_{2}$ is uniformly distributed over the interval $[5-\epsilon, 5+\epsilon]$ with $\epsilon$ arbitrarily small. If both users have the same average money constraint, two strategy pairs are given in Fig. 1(a), (b), (d), and (e). They are both Nash equilibrium strategy pairs by definition. Given user 2's strategy shown in Fig. 1(b), user 1 can bid anything less than $\tau$, which is the lowest bid of user 2, during the interval $[0, \sigma]$ since its throughput will be unaffected (this is the reason that multiple Nash equilibriums exist). In Fig. 1(a), we show the case that user 1 implements a strict linear bidding function, resulting in an expected throughput of 2.78 for user 1 and 3.33 for user 2 . Although user 1's bid during interval $[0, \sigma]$ will not change its own throughput, it will affect the amount of money user 2 has to pay (i.e., user 2 has to pay more to win a slot if user 1's bid is close to $\tau$ instead of 0 during $[0, \sigma]$; consequently, user 2 will have less money to bid in other slots). Thus, a rational decision for user 1 is not to bid anything less than the smallest bid of user 2 . Therefore, the Nash equilibrium strategy 


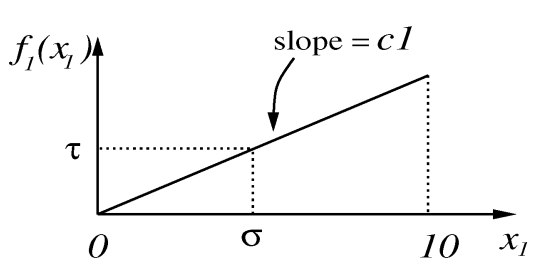

(a)

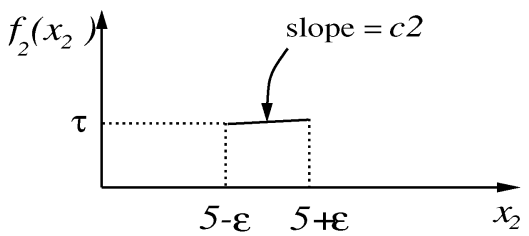

(b)

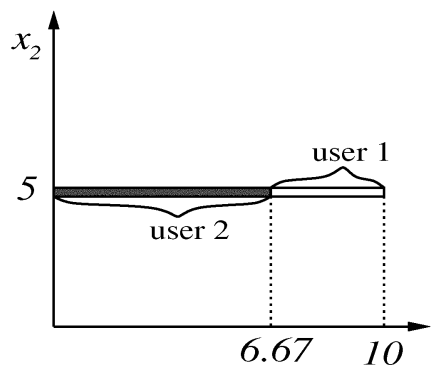

(c)

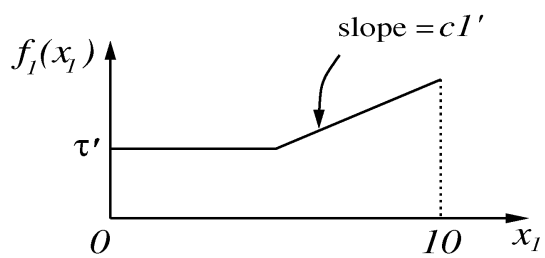

(d)

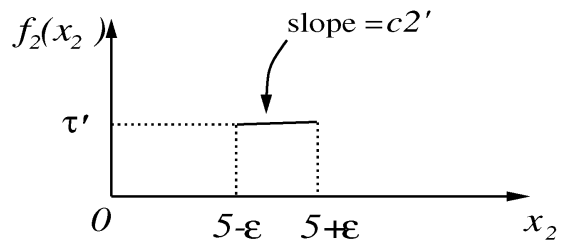

(e)

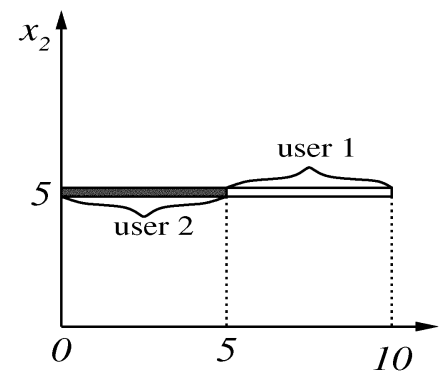

(f)

Fig. 1. (a) Bidding function for user 1 when using linear bidding function. (b) Bidding function for user 2. (c) Resulting allocation shown in the support of $X_{1}$ and $X_{2}$. (d) Bidding function for user 1 when it tries to make user 2 to pay more. (e) User 2's bidding function. (f) Resulting allocation when users using bidding function shown in (d) and (e).

pair shown in Fig. 1(d) and (e) is a more reasonable equilibrium strategy pair for this game. The outcome of the game is in this sense unique.

\section{Exponential Distribution}

When the channel state $X_{i}$ is exponentially distributed with rate $\mu_{i}$, the analysis in the general distribution section is still valid. The unique Nash equilibrium strategy pair has the same form as the uniform case: $f_{1}^{*}\left(x_{1}\right)=c_{1} \cdot x_{1}$ and $f_{2}^{*}\left(x_{2}\right)=c_{2} \cdot x_{2}$. Using (10) and (11), we get a relationship between $c_{1}$ and $c_{2}$ to be $c_{1} / c_{2}=\alpha_{1} \cdot \mu_{1} / \alpha_{2} \cdot \mu_{2}$. Thus, the optimal allocation is given by

$$
A^{*}\left(x_{1}, x_{2}\right)= \begin{cases}2, & \text { if } x_{2}>\left(\frac{c_{1}}{c_{2}}\right) x_{1} \\ 1, & \text { otherwise }\end{cases}
$$

Write the decision in another form $\mu_{2} X_{2}>\left(\alpha_{1} / \alpha_{2}\right) \mu_{1} X_{1}$. We see that only the normalized channel state distribution (i.e., $X_{2} / E\left[X_{2}\right]$ and $X_{1} / E\left[X_{1}\right]$, where $E\left[X_{2}\right]=1 / \mu_{2}, E\left[X_{1}\right]=$ $\left.1 / \mu_{1}\right)$ are used in the comparison. This result corroborates the score-based scheduler proposed by [18], which selects a user when its transmission rate is high relative to its own rate statistics. The expected throughput for each user is given by

$$
\begin{aligned}
& G_{1}=\frac{1}{\mu_{1}}\left[1-\frac{\alpha_{2}^{2}}{\left(\alpha_{1}+\alpha_{2}\right)^{2}}\right] \\
& G_{2}=\frac{1}{\mu_{2}}\left[1-\frac{\alpha_{1}^{2}}{\left(\alpha_{1}+\alpha_{2}\right)^{2}}\right] .
\end{aligned}
$$

\section{The N-Users Game}

In this section, we explore the Nash equilibrium of the second-price auction in which $N$ users, each with an average money constraint $\alpha_{i}$, compete for time slots. Given user $i$ 's strategy $f_{i} \in F_{i}$ with range from $f_{i}\left(l_{i}\right)=a_{i}$ to $f_{i}\left(u_{i}\right)=b_{i}$ for $i=2, \cdots, N$, user 1 wants to maximize its own expected throughput, while satisfying its expected money constraint. For a given $\left\{f_{2}, \cdots, f_{N}\right\}$, if user 1 chooses a bidding function $f_{1}$, the expected throughput or payoff function for user 1 is given by

$$
g_{1}=E_{X_{1}, X_{2}, \cdots, X_{N}}\left[X_{1} \cdot 1_{f_{1}\left(X_{1}\right) \geq \max \left\{f_{2}\left(X_{2}\right), \cdots, f_{N}\left(x_{N}\right)\right\}} .\right.
$$

The set of feasible bidding functions for user 1 , denoted here as $S_{1}\left(f_{2}, \cdots, f_{N}\right)$, can be written as

$$
\begin{gathered}
S_{1}\left(f_{2}, \cdots, f_{N}\right)=\left\{f_{1} \in F_{1} \mid E_{X_{2}, \cdots, X_{N}}\left[\operatorname { m a x } \left\{f_{2}\left(X_{2}\right), \cdots,\right.\right.\right. \\
\left.\left.f_{N}\left(x_{N}\right)\right\} \cdot 1_{f_{1}\left(X_{1}\right) \geq \max \left\{f_{2}\left(X_{2}\right), \cdots, f_{N}\left(x_{N}\right)\right\}} \leq \alpha_{1}\right\} .
\end{gathered}
$$

Similar to the two-users case, we define the inverse function as the following for $i=2, \cdots, N$ :

$$
h_{i}(y)= \begin{cases}l_{i}, & \text { if } y \leq a_{i} \\ f_{i}^{-1}(y), & \text { if } a_{i}<y<b_{i} . \\ u_{i}, & \text { if } y \geq b_{i}\end{cases}
$$

The following theorem characterizes the best response of user 1 for fixed $\left\{f_{2}, \cdots, f_{N}\right\}$ in this $N$-user game.

Theorem 4: Given fixed bidding functions $\left\{f_{2}, \cdots, f_{N}\right\}$ for user 2 to user $N$, and $a=\min \left\{f_{2}\left(x_{2}\right), \cdots, f_{N}\left(x_{N}\right)\right\}$ and $b=$ 
$\max \left\{f_{2}\left(x_{2}\right), \cdots, f_{N}\left(x_{N}\right)\right\}$ for $\left\{x_{2}, \cdots, x_{N}\right\} \in X_{2} \times \cdots \times$ $X_{N}$, user 1's best response has the following form:

$$
\begin{array}{lll}
f_{1}\left(x_{1}\right) \leq a, & \text { for } & x_{1} \in\left[l_{2}, \theta_{1}\right] \\
f_{1}\left(x_{1}\right)=c_{1} \cdot x_{1}, & \text { for } & x_{1} \in\left[\theta_{1}, \theta_{2}\right] \\
f_{1}\left(x_{1}\right) \geq b, & \text { for } & x_{1} \in\left[\theta_{2}, u_{2}\right]
\end{array}
$$

where $\theta_{1}, \theta_{2} \in\left[l_{2}, u_{2}\right]$ and $c_{2} \cdot \theta_{1}=a, c_{2} \cdot \theta_{2}=b$.

Proof: See the appendix.

Theorem 5: A Nash equilibrium exists in the second-price auction game $\Gamma=\left[N,\left\{S_{i}\right\},\left\{g_{i}(\cdot)\right\}\right]$ with $\left\{S_{i}\right\}$ and $\left\{g_{i}(\cdot)\right\}$ defined in (19) and (18), respectively.

Proof: We can then follow the steps in the two users case to show the existence of a Nash equilibrium. The analysis is omitted for brevity.

\section{NASh Equilibrium StRategy With MULTIPLE BIDDING FUNCTIONS}

In the previous section, we restricted the strategy space of each user to be a single bidding function. Specifically, once a user, say user 1 , chooses a function, say $f_{1}(\cdot)$, for its strategy, it bids an amount of money equal to $f_{1}\left(x_{1}\right)$ when it sees its channel condition is $X_{1}=x_{1}$. In other words, user 1 uses the same bidding function $f_{1}(\cdot)$ for all time slots. In this section, we will relax this single bidding function assumption, and investigate whether users have incentive to use different bidding function for different time slot (i.e., user 1 employs the bidding function $f_{1}^{(1)}(\cdot)$ for time slot 1 , and $f_{1}^{(2)}(\cdot)$ for time slot 2$)$ as long as their average constraint is not violated, and, given that users can choose multiple bidding functions, we explore whether the Nash equilibrium exists.

Again, for simplicity, we consider a two-users game where the user's channel state is uniformly distributed over $[0,1]$. Let $F_{1}$ and $F_{2}$ be, as before, the set of continuous, increasing, square integrable real-valued functions over the support of $X_{1}$ and $X_{2}$, respectively. Then, the strategy space for user 1 , say $S_{1}$, and user 2, say $S_{2}$, are defined as follows:

$$
\begin{aligned}
& S_{1}=\left\{f_{1}^{(1)}, \cdots, f_{1}^{(n)} \in F_{1} \mid \frac{1}{n} \sum_{i=1}^{n} E\left[f_{1}^{(i)}\left(X_{1}\right)\right]=\alpha_{1}\right\} \\
& S_{2}=\left\{f_{2}^{(1)}, \cdots, f_{2}^{(n)} \in F_{2} \mid \frac{1}{n} \sum_{i=1}^{n} E\left[f_{2}^{(i)}\left(X_{2}\right)\right]=\alpha_{2}\right\} .
\end{aligned}
$$

For each user, a strategy is a sequence of bidding functions $f^{(1)}, \cdots, f^{(n)}$. Without loss of generality, we restrict each user to have $n$ different bidding functions, where $n$ can be chosen as an arbitrarily large number. Note that users now choose a strategy for a block of $n$ time slots instead of just for a single time slot, one bidding function for each slot. In order to maximize the overall throughput (over infinite horizon), each user chooses bidding functions to maximize the expected total throughput over this block of $n$ slots. The term $E\left[f_{1}^{(i)}\left(X_{1}\right)\right]$ denotes the expected amount of money spent by user 1 if it uses bidding function $f_{1}^{(i)}$ for the $i$ th slot in the block. The strategy space discussed in the previous section can be considered to be a special class of strategies of $S_{1}$ and $S_{2}$ in which each user can use only a single bidding function. More specifically, set $f_{1}=f_{1}^{(1)}=\cdots=f_{1}^{(n)}$ and $f_{2}=f_{2}^{(1)}=\cdots=f_{2}^{(n)}$.

To choose a strategy (i.e., a sequence of bidding functions) from the strategy space $S_{1}$ or $S_{2}$, a user encounters two problems. First, it must decide how to allocate its money among these $n$ bidding functions so that the average money constraint is still satisfied. Second, once the money allocated to the $i$ th bidding function is specified, a user has to choose a bidding function for the $i$ th slot. The second problem is already solved in the previous section (see Theorem 3). In this section, we will focus on the first problem that a user encounters, specifically, the problem of how to allocate money between bidding functions, while satisfying the following condition: The total expected amount of money for the sequence of $n$ bidding functions is $n \cdot \alpha_{1}$ for user 1 and $n \cdot \alpha_{2}$ for user 2. For convenience, we let $\sigma=\alpha_{1}, \beta=\alpha_{2}$, and further denote $\sigma_{i}, \beta_{i}$ to be the average money allocated in slot $i$ for user 1 and user 2, respectively. The strategy space or possible actions that can be taken by users are given by the following:

$$
\begin{aligned}
& \hat{S}_{1}=\left\{\sigma_{1}, \cdots, \sigma_{n} \mid \sigma_{1}+\cdots+\sigma_{n}=n \cdot \sigma\right\} \\
& \hat{S}_{2}=\left\{\beta_{1}, \cdots, \beta_{n} \mid \beta_{1}+\cdots+\beta_{n}=n \cdot \beta\right\} .
\end{aligned}
$$

The objective of each user is still to maximize its own throughput. When user 1 and user 2 allocate $\sigma_{i}$ and $\beta_{i}$ for their $i$ th bidding function which is given in Theorem 3 , the payoff functions are $G_{1}\left(\sigma_{i}, \beta_{i}\right)$ for user 1 and $G_{2}\left(\sigma_{i}, \beta_{i}\right)$ for user 2 .

The following lemma gives us a Nash equilibrium strategy pair for the auction game described in this section.

Lemma 2: Given that user 2's strategy is to allocate its money evenly among its bidding functions (i.e., $\beta_{i}=\beta, i=1 \cdots n$ ), user 1 's best response is to allocate its money evenly as well (i.e., $\sigma_{i}=\sigma, i=1 \cdots n$ ); and vice versa. Therefore, a Nash equilibrium strategy pair for this auction is for both users to allocate their money evenly.

Proof: Without loss of generality, we consider the case that $n=2$ where each user's strategy can consist of two different bidding functions. Suppose that user 2 allocates $\beta$ for both bidding functions $f_{2}^{(1)}$ and $f_{2}^{(2)}$, and user 1 allocates $\sigma_{1}$ for bidding function $f_{1}^{(1)}$ and $\sigma_{2}$ for bidding function $f_{1}^{(2)}$, where $\sigma_{1}+\sigma_{2}=2 \sigma$ and $\sigma_{1} \neq \sigma_{2}$. We will show that the throughput for user $1 G_{1}\left(\sigma_{1}, \beta\right)+G_{1}\left(\sigma_{2}, \beta\right)$ is maximized when $\sigma_{1}=\sigma_{2}=\sigma$. Assume $\beta / 2<\sigma<\beta$. First, we consider the case that $\sigma_{1} \leq \beta$ and $\sigma_{2} \leq \beta$. The equation $G_{1}\left(\sigma_{1}, \beta\right)$ with $\beta$ fixed

$$
G_{1}\left(\sigma_{1}, \beta\right)=\frac{\sigma_{1}}{\beta+2 \sigma_{1}}
$$

becomes

$$
F(t)=\frac{t}{1+t}
$$

where $t=\sigma_{1} / \beta . F(t)$ is concave for $t \geq 0$. Thus, we have $G_{1}\left(\sigma_{1}, \beta\right)+G_{1}\left(\sigma_{2}, \beta\right)$ maximized when $\sigma_{1}=\sigma_{2}=\sigma$. For the case that $\sigma_{1} \geq \beta$ and $\sigma_{2}=2 \sigma-\sigma_{1} \leq \beta$, we have from (16) and (17)

$G_{1}\left(\sigma_{1}, \beta\right)+G_{1}\left(2 \sigma-\sigma_{1}, \beta\right)=\frac{1}{2}-\frac{3 \beta^{2}}{2\left(\sigma_{1}+2 \beta\right)^{2}}+\frac{2 \sigma-\sigma_{1}}{\beta+2\left(2 \sigma-\sigma_{1}\right)}$.

The above function can be shown to be strictly decreasing for $\sigma_{1} \in[\beta, 2 \sigma]$. Hence, it is optimal to choose $\sigma_{1}=\beta$ for $\sigma_{1}$ in 
the interval $[\beta, 2 \sigma]$. We also know that in the case $\sigma_{1} \leq \beta$ and $\sigma_{2} \leq \beta, G_{1}\left(\sigma_{1}, \beta\right)+G_{1}\left(\sigma_{2}, \beta\right)$ is maximized when $\sigma_{1}=\sigma_{2}=$ $\sigma$. Therefore, given user 2 allocates its money evenly among its bidding functions (i.e., $\beta_{i}=\beta, i=1 \cdots n$ ), user 1's best response is to allocate its money evenly as well.

We have already obtained a Nash equilibrium strategy pair from the above lemma. The following theorem states that this Nash equilibrium strategy pair is in fact unique within the strategy space considered.

Theorem 6: For the second-price auction with user's strategy space defined in (22), a unique Nash equilibrium strategy for both users is to allocate their money evenly among the bidding functions.

Proof: The complete proof is in the appendix.

In this section, users are given more freedom in choosing their strategies (i.e., they can choose $n$ different bidding functions). However, as Theorem 6 shows, the unique Nash equilibrium strategy pair is for each user to use a single bidding function from its strategy space. Thus, the throughput result obtained in this broader strategy space- $S_{1}$ and $S_{2}$ is the same as the throughput result from previous section. Therefore, there is no incentive for a user to use different bidding functions.

\section{Pareto Optimality of the Nash EQUILIBRIUM STRATEGIES}

Thus far, we have a Nash equilibrium strategy pair and the resulting throughput when both players choose to use the Nash equilibrium strategy. In this section, we want to address the question whether the allocation resulting from the Nash equilibrium strategy is efficient, or Pareto optimal. An allocation is said to be Pareto optimal if it is impossible to make some individuals better off without making some other individuals worse off. This concept is a formalization of the idea that there is no waste in the allocation process.

We start by investigating an allocation with a fairness constraint that requires the resulting throughput of the users to be kept at a constant ratio. Specifically, let $G_{1}$ and $G_{2}$ denote the expected throughput for user 1 and user 2, respectively. We have the following optimization problem: for some nonnegative $a$

$$
\begin{aligned}
& \max G_{1}+G_{2} \\
& \text { subj. } \frac{G_{1}}{G_{2}}=a .
\end{aligned}
$$

The optimal allocation is to divide the possible channel state realizations, $\left(x_{1}, x_{2}\right)$, into two regions by the separation line $x_{2}=c \cdot x_{1}$, where $c$ is some positive real number. Above the line (i.e., $x_{2}>c \cdot x_{1}$ ), the transmitter will assign the slot to user 2. Below the line (i.e., $x_{2}<c \cdot x_{1}$ ), the transmitter will assign the slot to user 1.

To prove the above, we use a method that is similar to the one in [9]. By using an allocation $A$, the resulting throughput for user 1 and user 2 are $G_{1}^{A}=E\left[X_{1} \cdot 1_{A\left(X_{1}, X_{2}\right)=1}\right]$ and $G_{2}^{A}=$ $E\left[X_{2} \cdot 1_{A\left(X_{1}, X_{2}\right)=2}\right]$, respectively. Now, we define an allocation as follows:

$$
A^{*}\left(x_{1}, x_{2}\right)= \begin{cases}1, & \text { if } x_{1}\left(1+\lambda^{*}\right) \geq x_{2}\left(1-a \cdot \lambda^{*}\right) \\ 2, & \text { otherwise }\end{cases}
$$

where $\lambda^{*}$ is chosen such that $G_{1}^{A^{*}} / G_{2}^{A^{*}}=a$ is satisfied.
Consider an arbitrary allocation $A$ that satisfies $G_{1}^{A} / G_{2}^{A}=a$. We have

$$
\begin{aligned}
E[ & \left.X_{1} \cdot 1_{A\left(X_{1}, X_{2}\right)=1}\right]+E\left[X_{2} \cdot 1_{A\left(X_{1}, X_{2}\right)=2}\right] \\
= & E\left[X_{1} \cdot 1_{A\left(X_{1}, X_{2}\right)=1}\right]+E\left[X_{2} \cdot 1_{A\left(X_{1}, X_{2}\right)=2}\right] \\
& +\lambda^{*}\left(E\left[X_{1} \cdot 1_{A\left(X_{1}, X_{2}\right)=1}\right]\right. \\
& \left.\quad-a E\left[X_{2} \cdot 1_{A\left(X_{1}, X_{2}\right)=2}\right]\right) \\
= & E\left[\left(X_{1}+\lambda^{*} X_{1}\right) \cdot 1_{A\left(X_{1}, X_{2}\right)=1}\right] \\
& +E\left[\left(X_{2}-a \lambda^{*} X_{2}\right) \cdot 1_{A\left(X_{1}, X_{2}\right)=2}\right] \\
\leq & E\left[\left(X_{1}+\lambda^{*} X_{1}\right) \cdot 1_{A^{*}\left(X_{1}, X_{2}\right)=1}\right] \\
& +E\left[\left(X_{2}-a \lambda^{*} X_{2}\right) \cdot 1_{A^{*}\left(X_{1}, X_{2}\right)=2}\right] \\
= & E\left[X_{1} \cdot 1_{A^{*}\left(X_{1}, X_{2}\right)=1}\right]+E\left[X_{2} \cdot 1_{A^{*}\left(X_{1}, X_{2}\right)=2}\right] \\
& +\lambda^{*}\left(E\left[X_{1} \cdot 1_{A^{*}\left(X_{1}, X_{2}\right)=1}\right]\right. \\
& \left.\quad-a E\left[X_{2} \cdot 1_{A^{*}\left(X_{1}, X_{2}\right)=2}\right]\right) \\
= & E\left[X_{1} \cdot 1_{A^{*}\left(X_{1}, X_{2}\right)=1}\right]+E\left[X_{2} \cdot 1_{A^{*}\left(X_{1}, X_{2}\right)=2}\right] .
\end{aligned}
$$

The inequality in the middle is from the definition of $A^{*}$. Specifically, if we were asked to choose an allocation $A$ to maximize $E\left[\left(X_{1}+\lambda^{*} X_{1}\right) \cdot 1_{A\left(X_{1}, X_{2}\right)=1}\right]+E\left[\left(X_{2}-\right.\right.$ $\left.a \lambda^{*} X_{2}\right) \cdot 1_{A\left(X_{1}, X_{2}\right)=2}$. Then, $A^{*}$ will be an optimal scheme from its definition. Thus, $A^{*}\left(X_{1}, X_{2}\right)$ is an optimal solution to the optimization problem in (23).

So far, we have shown that the optimal allocation for the problem in (23) has the same form as the allocation scheme resulted from the Nash equilibrium strategy of second-price auction (i.e., both allocation schemes compare channel state realization $x_{1}$ with $c \cdot x_{2}$ where $c$ is a constant). Examining the optimization problem in (23), we see that the resulting throughput obtained is Pareto optimal. To show this, suppose $G_{1}^{p}$ and $G_{2}^{p}$ are the throughput of a Pareto optimal allocation, and $G_{1}^{p} / G_{2}^{p}=c_{p}$. If the optimal solution of the problem maximizing $G_{1}+G_{2}$ subject to the constraint $G_{1} / G_{2}=c_{p}$ are $G_{1}^{*}$ and $G_{2}^{*}$, we must have $G_{1}^{*}+G_{2}^{*} \geq G_{1}^{p}+G_{2}^{p}$ which implies $G_{1}^{*} \geq G_{1}^{p}$ and $G_{2}^{*} \geq G_{2}^{p}$ since $G_{1}^{p} / G_{2}^{p}=c_{p}$ and $G_{1}^{*} / G_{2}^{*}=c_{p}$. From the assumption that $G_{1}^{p}$ and $G_{2}^{p}$ are the throughput of a Pareto optimal allocation, we must have $G_{1}^{*}=G_{1}^{p}$ and $G_{2}^{*}=G_{2}^{p}$. Therefore, the solution to the optimization problem (23) is Pareto optimal which also implies the Pareto optimality of the allocation resulting from equilibrium strategy since they have the same form.

\section{COMPARISON With Other ALLOCATION SCHEMES}

Based on our previous analysis on the Nash equilibrium strategy of the second-price auction with average money constraint, we can implement a centralized opportunistic scheduler that is fair and efficient. Instead of allowing users to actually bid for each time slot, the centralized scheduler will assign time slots according to the Nash equilibrium strategy based on users' average money amount. If users are assumed to have equal priority (as in the cases of maxmin fairness and proportion fairness), the scheduler simply let each user have an equal money constraint, and assigns time slots according to the equilibrium 
strategy. Later in this section, we will compare our centralized scheduling scheme with proportional fair scheduling scheme. But first, we need to quantify the loss of efficiency by using Nash equilibrium strategies. Due to the fairness constraint, total system throughput will decrease as compared with the maximum throughput attainable without any fairness constraint. Hence, we would like to compare the total throughput of the Nash equilibrium strategy to that of an unconstrained strategy. We address this question by first considering an allocation that maximizes total throughput subject to no constraint.

\section{A. Maximizing Throughput With No Constraint}

To maximize throughput without any constraints, the transmitter serves the user with a better channel state during each time slot. Then, the expected throughput is $E\left[\max \left\{X_{1}, X_{2}\right\}\right]$. For $X_{1}$ and $X_{2}$ independent uniformly distributed in $[0,1]$, we have $E\left[\max \left\{X_{1}, X_{2}\right\}\right]=2 / 3$. Using the Nash equilibrium strategy, the total expected system throughput, $G_{1}+G_{2}$, is $1 / 2$ in the worst case (i.e., one users gets all of the time slots, while the other user is starving). Thus, the channel allocation proposed here can achieve at least $75 \%$ of the maximum attainable throughput. This gives us a lower bound of the throughput performance of the allocation derived from the Nash equilibrium pair.

\section{B. Proportional Fairness}

In this section, we examine the well-known proportional fairness allocation. Let $G_{1}, G_{2}, A$ be defined similarly as in the previous section. The objective of proportional fairness is to maximize the term $\left(\log G_{1}+\log G_{2}\right)$ [4]. Specifically, the optimization problem is given by

$$
\begin{aligned}
\max _{A} & \log E\left[X_{1} \cdot 1_{A\left(X_{1}, X_{2}\right)=1}\right]+\log E\left[X_{2} \cdot 1_{A\left(X_{1}, X_{2}\right)=2}\right] \\
= & \max _{A} \log \left[\int_{\left(x_{1}, x_{2}\right) \mid A\left(x_{1}, x_{2}\right)=1} x_{1} p_{X_{1}}\left(x_{1}\right) p_{X_{2}}\left(x_{2}\right) d x_{1} d x_{2}\right] \\
& +\log \left[\int_{\left(x_{1}, x_{2}\right) \mid A\left(x_{1}, x_{2}\right)=2} x_{2} p_{X_{1}}\left(x_{1}\right) p_{X_{2}}\left(x_{2}\right) d x_{1} d x_{2}\right] .
\end{aligned}
$$

It is straightforward to see that the optimal allocation policy has to be a threshold rule. That is, for given $\left(\bar{x}_{1}, \bar{x}_{2}\right)$ on the threshold and a particular time slot with channel state $\left(x_{1}, x_{2}\right)$, the scheduler will assign the time slot to user 1 if the channel state pair $\left(x_{1}, x_{2}\right) \in\left\{(a, b) \mid a>\bar{x}_{1}\right.$ and $\left.b<\bar{x}_{2}\right\}$, and to user 2 if $\left(x_{1}, x_{2}\right) \in\left\{(a, b) \mid a<\bar{x}_{1}\right.$ and $\left.b>\bar{x}_{2}\right\}$. To get the optimal allocation policy, we consider again a point $\left(\bar{x}_{1}, \bar{x}_{2}\right)$ on the threshold and a small region with probability $\Delta$ around that point. Intuitively, since this region is on the threshold, an optimal scheduler can allocate it to either user 1 or user 2 . If allocating the small region to user 1 will result in more gain than allocating

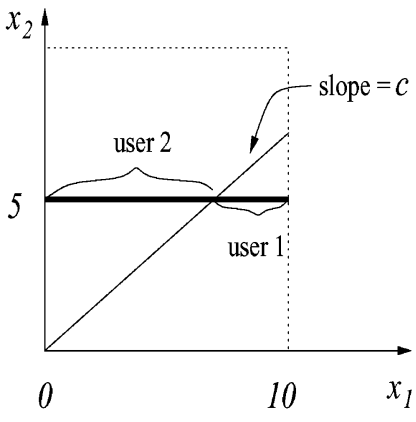

(a)

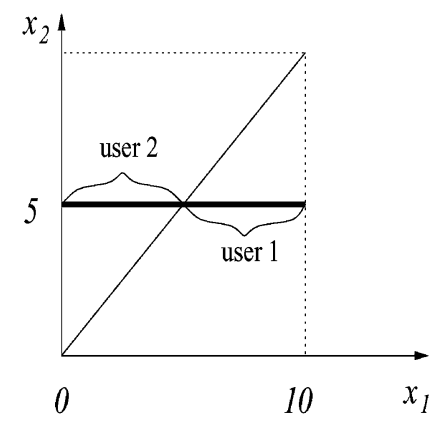

(b)
Fig. 2. (a) The proportional fair allocation scheme. (b) The second-price auction scheme with equal money constraint.

it to user 2, this region will not be on threshold anymore but belongs to user 1 . Thus, for $A^{*}$ to be an optimal allocation rule, we have the following first-order approximation:

$$
\begin{gathered}
\log \left[\int_{\left(x_{1}, x_{2}\right) \mid A^{*}\left(x_{1}, x_{2}\right)=1} x_{1} p_{X_{1}}\left(x_{1}\right) p_{X_{2}}\left(x_{2}\right) d x_{1} d x_{2}+\bar{x}_{1} \cdot \Delta\right] \\
-\log \left[\int_{\left(x_{1}, x_{2}\right) \mid A^{*}\left(x_{1}, x_{2}\right)=1} x_{1} p_{X_{1}}\left(x_{1}\right) p_{X_{2}}\left(x_{2}\right) d x_{1} d x_{2}\right] \\
=\frac{\bar{x}_{1} \cdot \Delta}{\int_{\left(x_{1}, x_{2}\right) \mid A^{*}\left(x_{1}, x_{2}\right)=1} x_{1} p_{X_{1}}\left(x_{1}\right) p_{X_{2}}\left(x_{2}\right) d x_{1} d x_{2}} \cdot
\end{gathered}
$$

Similar equation can be written for $\bar{x}_{2}$. Combine both equations, we have the following that describes the threshold of $A^{*}$ :

$$
\frac{\bar{x}_{1}}{G_{1}^{A^{*}}}=\frac{\bar{x}_{2}}{G_{2}^{A^{*}}} .
$$

The optimal allocation can then be stated as

$$
A^{*}\left(x_{1}, x_{2}\right)= \begin{cases}1, & \text { if } x_{1} \geq c \cdot x_{2} \\ 2, & \text { otherwise }\end{cases}
$$

where the constant $c=G_{1}^{A^{*}} / G_{2}^{A^{*}}$. We find the allocation with proportional fairness criteria has the same form as the allocation that resulted from the Nash equilibrium strategy (i.e., both of them are straight lines). Therefore, it is interesting to compare the performance of the proportional fairness algorithm to that of the auction algorithm. Consider an example where $X_{1}$ is uniformly distributed over the interval $[0,10]$ and $X_{2}$ is uniformly distributed over the interval $[5-\epsilon, 5+\epsilon]$ (consistent with our previous example). Assuming $\epsilon$ is small, we can treat $X_{2}$ as a constant. Using the proportional fairness scheme, we need to find a threshold $c$ such that $x_{2}=c \cdot x_{1}$ and $c=G_{1} / G_{2}$. From Fig. 2(a), we see that $G_{2}=(5 / 10 c) \cdot 5$ and $G_{1}=$ $((10+5 / c) / 2)(10-5 / c) \cdot(1 / 10)$. Setting $G_{2} / G_{1}=c$, we have $c=1 / \sqrt{2}=0.707$. As a result of the proportional fair algorithm, the scheduler will assign almost $71 \%$ of the time slots to 
user 2. The user with a constant channel states obviously benefits more from the proportional fairness algorithm. For comparison, we use the centralized scheduler (based on the auction algorithm) described in the early part of this section (i.e., we let each user have the same average money constraint when employing the second-price auction algorithm). From Fig. 2(b), we see that both users get almost half of the time slots (it does not bias toward user with a constant channel state). Furthermore, it also results in a higher total system throughput than that of the proportion fairness scheme. Specifically, the auction scheme yields a total throughput of 6.25 , while the proportional fairness scheme yields a total throughput of 6.0.

\section{CONCLUSION}

We apply an auction algorithm to the problem of fair allocation of a wireless fading channel. Using the second-price auction mechanism, we are able to obtain the Nash equilibrium strategies for general channel state distribution. Our strategy allocates bandwidth to the users in accordance with the amount of money that they possess. Hence, this scheme can be viewed as a mechanism for providing QoS differentiation; whereby users are given fictitious money that they can use to bid for the channel. By allocating users different amounts of money, the resulting QoS differentiation can be achieved.

In this paper, we find the unique Nash equilibrium strategy for certain commonly used channel state distribution. We also show that the Nash equilibrium strategy of this auction leads to an allocation at which total throughput is no worse than $3 / 4$ the maximum possible throughput when fairness constraints are not imposed (i.e., slots are allocated to the user with the better channel) under uniform distribution. Moreover, the equilibrium strategies leads to an allocation that is Pareto optimal. Based on the Nash equilibrium strategies of the second-price auction with money constraint, we also propose a centralized opportunistic scheduler that does not suffer the shortcomings associated with the proportional fair and the time fraction fair scheduler. However, the problem of how to obtain the multiplicative constant in user's equilibrium bidding strategy using a computational efficient way has yet to be explored. Also, to make our proposed centralized scheduler (based on the Nash equilibrium strategy) suitable for real-time implementation, an algorithm that does not require the prior knowledge of channel distribution but still results in the Nash equilibrium allocation for each user will be an important topic for the future research.

\section{APPENDIX}

\section{Proof of Theorem 4:}

Proof: If user 1 bids $y$ for a particular time slot, the probability that it win, denoted as $P_{1}^{\text {win }}(y)$, is given by

$$
\begin{aligned}
P_{1}^{\text {win }}(y) & =\operatorname{Pr}\left(\max \left\{f_{2}\left(X_{2}\right), \cdots, f_{N}\left(x_{N}\right)\right\} \leq y\right) \\
& =\operatorname{Pr}\left(f_{2}\left(X_{2}\right) \leq y, \cdots, f_{N}\left(X_{N}\right) \leq y\right)
\end{aligned}
$$

The optimization for user 1 can be written as follows:

$$
\begin{gathered}
\max _{f_{1}} \int_{0}^{1} \int_{l_{2}}^{h_{2}\left(f_{1}\left(x_{1}\right)\right)} \cdots \int_{l_{N}}^{h_{N}\left(f_{1}\left(x_{1}\right)\right)} x_{1} \\
\cdot p_{X_{1}}\left(x_{1}\right) \cdots p_{X_{N}}\left(x_{N}\right) d x_{1} \cdots d x_{N}
\end{gathered}
$$

subject to

$$
\begin{gathered}
\int_{0}^{1} \int_{l_{2}}^{h_{2}\left(f_{1}\left(x_{1}\right)\right)} \cdots \int_{l_{N}}^{h_{N}\left(f_{1}\left(x_{1}\right)\right)} \max \left\{f_{2}\left(x_{2}\right), \cdots, f_{N}\left(x_{N}\right)\right\} \\
\cdot p_{X_{1}}\left(x_{1}\right) \cdots p_{X_{N}}\left(x_{N}\right) d x_{1} \cdots d x_{N} \leq \alpha_{1} .
\end{gathered}
$$

After writing the Lagrangian function, we then solve the following optimization problem:

$$
\begin{aligned}
& \max _{f_{1}\left(x_{1}\right)} \int_{l_{2}}^{h_{2}\left(f_{1}\left(x_{1}\right)\right)} \cdots \\
& \quad \int_{l_{N}}^{h_{N}\left(f_{1}\left(x_{1}\right)\right)}\left(x_{1}-\lambda_{1} \max \left\{f_{2}\left(x_{2}\right), \cdots, f_{N}\left(x_{N}\right)\right\}\right) \\
& \quad \cdot p_{X_{2}}\left(x_{2}\right) \cdots p_{X_{N}}\left(x_{N}\right) d x_{2} \cdots d x_{N} .
\end{aligned}
$$

Writing $y=f_{1}\left(x_{1}\right)$ for convenience, we have the following:

$$
\begin{array}{r}
\max _{y} \int_{l_{2}}^{h_{2}(y)} \cdots \int_{l_{N}}^{h_{N}(y)}\left(x_{1}-\lambda_{1} \max \left\{f_{2}\left(x_{2}\right), \cdots, f_{N}\left(x_{N}\right)\right\}\right) \\
\cdot p_{X_{2}}\left(x_{2}\right) \cdots p_{X_{N}}\left(x_{N}\right) d x_{2} \cdots d x_{N} .
\end{array}
$$

The term $\left(x_{1}-\lambda_{1} \max \left\{f_{2}\left(x_{2}\right), \cdots, f_{N}\left(x_{N}\right)\right\}\right)$ is decreasing since $\left(\lambda_{1} \max \left\{f_{2}\left(x_{2}\right), \cdots, f_{N}\left(x_{N}\right)\right\}\right)$ is increasing. Therefore, it is desirable to choose $y$ as large as possible, while keeping $\left(x_{1}-\lambda_{1} \max \left\{f_{2}\left(x_{2}\right), \cdots, f_{N}\left(x_{N}\right)\right\}\right) \geq 0$.

For a fixed $x_{1}$, if the term $\left(x_{1}-\right.$ $\left.\lambda_{1} \max \left\{f_{2}\left(x_{2}\right), \cdots, f_{N}\left(x_{N}\right)\right\}\right) \quad$ is positive for all $x_{i} \in\left[l_{i}, u_{i}\right]$ for $i=2, \cdots, N$, the optimal $y^{*}$ can be chosen such that $y^{*} \geq \max \left\{b_{2}, \cdots, b_{N}\right\}$. Likewise, if the term $x_{1}-\lambda_{1} \max \left\{f_{2}\left(x_{2}\right), \cdots, f_{N}\left(x_{N}\right)\right\}$ is negative for all $x_{i} \in\left[l_{i}, u_{i}\right]$ for $i=2, \cdots, N$, the optimal $y^{*}$ can be chosen such that $y^{*} \leq \min \left\{a_{2}, \cdots, a_{N}\right\}$. In the case that $\left(x_{1}-\lambda_{1} \max \left\{f_{2}\left(x_{2}\right), \cdots, f_{N}\left(x_{N}\right)\right\}\right)=0$ for some $\left(x_{2}, \cdots, x_{N}\right) \in\left[l_{2}, u_{2}\right] \times \cdots \times\left[l_{N}, u_{N}\right]$, we can choose $y^{*}$ such that $\left(x_{1}-\lambda_{1} \max \left\{f_{2}\left(h_{2}\left(y^{*}\right)\right), \cdots, f_{N}\left(h_{N}\left(y^{*}\right)\right)\right\}\right)=0$. From the definition of $h_{i}(\cdot)$ in $(20)$, each term $f_{i}\left(h_{i}(y)\right)$ equals to $\min \left\{y^{*}, b_{i}\right\}$ for $i=2, \cdots, N$. Hence, we have the following:

$$
\max \left\{f_{2}\left(h_{2}\left(y^{*}\right)\right), \cdots, f_{N}\left(h_{N}\left(y^{*}\right)\right)\right\}=y^{*} .
$$

Consequently, the optimal bid for user $1, y^{*}$, is $\left(1 / \lambda_{1}\right) x_{1}$. Again, the optimal bidding strategy for a user in the $\mathrm{N}$-user game is a linear function of the user's channel state. The constant coefficient, i.e., $1 / \lambda_{1}$, is chosen such that the average money constraint is met with equality.

\section{Proof of Theorem 6:}

Proof: Again, we consider $n=2$ case for simplicity. For $\sigma_{1}+\sigma_{2}=2 \sigma$ and $\beta_{1}+\beta_{2}=2 \beta$, this theorem stated that the pair $\left(\sigma_{1}, \beta_{1}\right)$ and $\left(\sigma_{2}, \beta_{2}\right)$ cannot be in equilibrium if $\sigma_{1} \neq \sigma_{2}$ and $\beta_{1} \neq \beta_{2}$. We will show this by contradiction. Here, we present the proof for the case that $\sigma_{1} \leq \beta_{1}$ and $\sigma_{1} \leq \beta_{1}$. Other 
cases can be shown similarly. Now, suppose the pair $\left(\sigma_{1}, \beta_{1}\right)$ and $\left(\sigma_{2}, \beta_{2}\right)$ are in equilibrium for $\sigma_{1} \neq \sigma_{2}$ and $\beta_{1} \neq \beta_{2}$. That is, for given $\beta_{1}$ and $\beta_{2}, \sigma_{1}$ and $\sigma_{2}$ are chosen such that user 1 's throughput $G_{1}\left(\sigma_{1}, \beta_{1}\right)+G_{1}\left(\sigma_{2}, \beta_{2}\right)$ is the maximum. This implies the following:

$$
\left.\frac{\partial G_{1}\left(\sigma, \beta_{1}\right)}{\partial \sigma}\right|_{\sigma=\sigma_{1}}=\left.\frac{\partial G_{1}\left(\sigma, \beta_{2}\right)}{\partial \sigma}\right|_{\sigma=\sigma_{2}} .
$$

To see this, if $\left.\left(\partial G_{1}\left(\sigma, \beta_{1}\right) / \partial \sigma\right)\right|_{\sigma=\sigma_{1}}>\left(\partial G_{1}\left(\sigma, \beta_{2}\right) /\right.$ $\partial \sigma)\left.\right|_{\sigma=\sigma_{2}}$, we will have $G_{1}\left(\sigma_{1}+\delta, \beta_{1}\right)+G_{1}\left(\sigma_{2}-\delta, \beta_{2}\right)>$ $G_{1}\left(\sigma_{1}, \beta_{1}\right)+G_{1}\left(\sigma_{2}, \beta_{2}\right)$ by first-order expansion, thus contradicting the statement that $G_{1}\left(\sigma_{1}, \beta_{1}\right)+G_{1}\left(\sigma_{2}, \beta_{2}\right)$ is the maximum throughput for user 1 for given $\beta_{1}$ and $\beta_{2}$.

Similarly, for given $\sigma_{1}$ and $\sigma_{2}$, if $\beta_{1}$ and $\beta_{2}$ maximize $G_{2}\left(\sigma_{1}, \beta_{1}\right)+G_{2}\left(\sigma_{2}, \beta_{2}\right)$, then

$$
\left.\frac{\partial G_{2}\left(\sigma_{1}, \beta\right)}{\partial \beta}\right|_{\beta=\beta_{1}}=\left.\frac{\partial G_{2}\left(\sigma_{2}, \beta\right)}{\partial \beta}\right|_{\beta=\beta_{2}} .
$$

By taking the derivative of (16) and (17), we get the following:

$$
\begin{aligned}
& \left.\frac{\partial G_{1}\left(\sigma, \beta_{1}\right)}{\partial \sigma}\right|_{\sigma=\sigma_{1}}=\frac{\beta_{1}}{\left(\beta_{1}+2 \sigma_{1}\right)^{2}} \\
& \left.\frac{\partial G_{2}\left(\sigma_{1}, \beta\right)}{\partial \beta}\right|_{\beta=\beta_{1}}=\frac{3 \sigma_{1}^{2}}{\left(\beta_{1}+2 \sigma_{1}\right)^{3}} .
\end{aligned}
$$

Substituting (31) into (29) and (32) into (30), we then have the following after combining (29) and (30):

$$
\begin{aligned}
\frac{\beta_{1}}{\beta_{2}} & =\frac{\left(\beta_{1}+2 \sigma_{1}\right)^{2}}{\left(\beta_{2}+2 \sigma_{2}\right)^{2}} \\
\frac{3 \sigma_{1}^{2}}{3 \sigma_{2}^{2}} & =\frac{\left(\beta_{1}+2 \sigma_{1}\right)^{3}}{\left(\beta_{2}+2 \sigma_{2}\right)^{3}} \\
\Rightarrow \frac{\sigma_{1}}{\beta_{1}^{\frac{3}{4}}} & =\frac{\sigma_{2}}{\beta_{2}^{\frac{3}{4}}} .
\end{aligned}
$$

Now, we have $\left(\sigma_{1} / \beta_{1}^{3 / 4}\right)=\left(\sigma_{2} / \beta_{2}^{3 / 4}\right)$. We further show that $\sigma_{1}=\sigma_{2}$ and $\beta_{1}=\beta_{2}$. Observe that for fixed $\beta_{1}$ and $\beta_{2}$, we can write

$$
G_{1}\left(\sigma, \beta_{1}\right)=\frac{\sigma}{\beta_{1}+2 \sigma}=\frac{\frac{\sigma}{\beta_{1}^{\frac{3}{4}}}}{\frac{\beta_{1}}{\beta_{1}^{\frac{3}{4}}}+\frac{2 \sigma}{\beta_{1}^{\frac{3}{4}}}} \triangleq F\left(\frac{\sigma}{\beta_{1}}\right)
$$

where

$$
F(\gamma)=\frac{\gamma}{\beta_{1}^{\frac{1}{4}}+2 \gamma}
$$

Thus, we have

$$
\begin{aligned}
& \left.\frac{\partial G_{1}\left(\sigma, \beta_{1}\right)}{\partial \sigma}\right|_{\sigma=\sigma_{1}}=\left.\left.\frac{1}{\beta_{1}^{\frac{3}{4}}} \frac{\partial F(\gamma)}{\partial \gamma}\right|_{\gamma=\frac{\sigma_{1}}{\beta_{1}^{\frac{3}{4}}}}\right|_{\sigma=\sigma_{2}}=\left.\frac{1}{\beta_{2}^{\frac{3}{4}}} \frac{\partial F(\gamma)}{\partial \gamma}\right|_{\gamma=\frac{\sigma_{2}}{\beta_{2}^{\frac{3}{4}}}}
\end{aligned}
$$

From (29), we have

$$
\left.\frac{1}{\beta_{1}^{\frac{3}{4}}} \frac{\partial F(\gamma)}{\partial \gamma}\right|_{\gamma=\frac{\sigma_{1}}{\beta_{1}^{\frac{3}{4}}}}=\left.\frac{1}{\beta_{2}^{\frac{3}{4}}} \frac{\partial F(\gamma)}{\partial \gamma}\right|_{\gamma=\frac{\sigma_{2}}{\beta_{2}^{\frac{3}{4}}}}
$$

It is easy to verify that $(\partial F(\gamma) / \partial \gamma) \neq 0 \forall \gamma \geq 0$. Therefore, since $\left(\sigma_{1} / \beta_{1}^{3 / 4}\right)=\left(\sigma_{2} / \beta_{2}^{3 / 4}\right)$, the above equation implies that $\beta_{1}=\beta_{2}$ which contradicts our original assumption of $\beta_{1} \neq \beta_{2}$. Therefore, the pair $\left(\sigma_{1}, \beta_{1}\right)$ and $\left(\sigma_{2}, \beta_{2}\right)$ cannot be in equilibrium if $\sigma_{1} \neq \sigma_{2}$ and $\beta_{1} \neq \beta_{2}$.

\section{ACKNOWLEDGMENT}

The authors gratefully acknowledge the helpful discussions with Prof. R. Johari, Prof. E. Altman, and Prof. A. Ozdaglar.

\section{REFERENCES}

[1] A. Fu, E. Modiano, and J. Tsitsiklis, "Optimal energy allocation for delay-constrained data transmission over a time-varying channel," in Proc. IEEE INFOCOM 2003, San Francisco, CA, Apr. 2003, pp. 1095-1105.

[2] P. Marbach and R. Berry, "Downlink resource allocation and pricing for wireless networks," in Proc. IEEE INFOCOM 2002, New York, Jun. 2002, pp. 1470-1479.

[3] P. Marbach, "Priority service and max-min fairness," in Proc. IEEE INFOCOM 2002, New York, Jun. 2002, pp. 266-275.

[4] P. Viswanath, D. Tse, and R. Laroia, "Opportunistic beamforming using dumb antennas," IEEE Trans. Inf. Theory, vol. 48, no. 6, pp. 1277-1294, Jun. 2002.

[5] L. Tassiulas and S. Sarkar, "Maxmin fair scheduling in wireless networks," in Proc. IEEE INFOCOM, New York, Jun. 2002, pp. 763-772.

[6] F. P. Kelly, A. K. Maulloo, and D. K. H. Tan, "Rate control for communication networks: shadow prices, proportional fairness and stability," $J$. Oper. Res. Soc., vol. 49, pp. 237-252, 1998.

[7] A. El Gamal, E. Uysal, and B. Prabhakar, "Energy-efficient transmission over a wireless link via lazy packet scheduling," in Proc. IEEE INFOCOM, Anchorage, AK, Apr. 2001, pp. 386-394.

[8] B. Collins and R. Cruz, "Transmission policies for time varying channels with average delay constraints," in Proc. Allerton Conf. on Commun. Control, and Comput., Monticello, IL, 1999.

[9] X. Liu, E. K. P. Chong, and N. B. Shroff, "Opportunistic transmission scheduling with resource-sharing constraints in wireless networks," IEEE J. Sel. Areas Commun., vol. 19, no. 10, pp. 2053-2064, Oct. 2001.

[10] D. Bertsekas and R. Gallager, Data Networks. Englewood Cliffs, NJ: Prentice-Hall, 1991.

[11] D. Bertsekas, Nonlinear Programming. Belmont, MA: Athena Scientific, 1999.

[12] R. Johari and J. Tsitsiklis, Efficiency loss in a network resource allocation game, Math. Oper. Res., pp. 407-435, 2004.

[13] P. Klemperer, "Auction theory: A guide to the literature," J. Economics Surveys, vol. 13, no. 3, pp. 227-286, Jul. 1999.

[14] Y.-K. Che and I. Gale, "Standard auctions with financially constrained bidders," Re. Economic Studies, vol. 65, pp. 1-21, Jan. 1998.

[15] T. R. Palfrey, "Multiple-object, discriminatory auctions with bidding constraints: A game-theoretic analysis," Manage. Sci., vol. 26, pp. 935-946, Sep. 1980.

[16] D. Famolari, N. Mandayam, and D. Goodman, "A new framework for power control in wireless data networks: Games, utility, and pricing," in Proc. Allerton Conf. Commun., Control, and Comput., Monticello, IL, Sep. 1998.

[17] T. Basar and R. Srikant, "Revenue-maximizing pricing and capacity expansion in a many-users regime," in Proc. IEEE INFOCOM, vol. 1, Jun. 2002, pp. 23-27.

[18] T. Bonald, "A score-based opportunistic scheduler for fading radio channels," in Proc. 5th Eur. Wireless Conf., Feb. 2004.

[19] A. Mas-Colell, M. D. Whinston, and J. R. Green, Microeconomic Theory. Oxford, U.K.: Oxford Univ. Press, 1995.

[20] K. C. Border, Fixed Point Theorems With Applications to Economics and Game Theory. Cambridge, U.K.: Cambridge Univ. Press, 1985. 


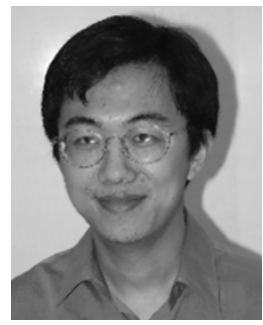

Jun Sun received the B.S. degree in computer engineering from University of Florida, Gainesville, in 1997 and the M.S. in electrical engineering from Massachusetts Institute of Technology (MIT), Cambridge, in 2002. He is currently working towards the Ph.D. degree in the Laboratory for Information and Decision Systems, MIT.

His research interest is on communication networks with emphasis on satellite and wireless networks.

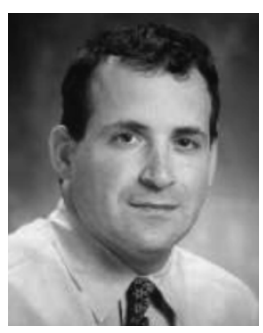

Eytan Modiano (S'90-M'93-SM'00) received the B.S. degree in electrical engineering and computer science from the University of Connecticut, Storrs, in 1986, and the M.S. and Ph.D. degrees from the University of Maryland, College Park, in 1989 and 1992, respectively, both in electrical engineering.

He was a Naval Research Laboratory Fellow from 1987 and 1992 and a National Research Council Post Doctoral Fellow during 1992-1993. From 1993 to 1999, he was with MIT Lincoln Laboratory, where he was the Project Leader for MIT Lincoln Laboratory's Next-Generation Internet (NGI) Project. Since 1999, he has been an Associate Professor in the Department of Aeronautics and Astronautics and the Laboratory for Information and Decision Systems (LIDS), MIT. His research is on communication networks and protocols with emphasis on satellite, wireless, and optical networks.

Dr. Modiano is currently an Associate Editor for Communication Networks for the IEEE TRANSACTIONS ON INFORMATION THEORY and for the International Journal of Satellite Communications. He had served as a Guest Editor for the IEEE JOURNAL ON SELECTED AREAS IN COMMUNICATIONS (Special Issue on WDM Network Architectures), the Computer Networks Journal (Special Issue on Broadband Internet Access), the Journal of Communications and Networks (Special Issue on Wireless Ad Hoc Networks), and for the IEEE JOURNAL OF LightwAVE TECHNOLOGY (Special Issue on Optical Networks). He is the Technical Program Co-Chair for WiOpt 2006 and INFOCOM 2007.

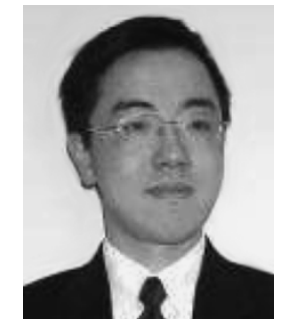

Lizhong Zheng (M'02) received the B.S. and M.S. degrees from the Department of Electronic Engineering, Tsinghua University, Tsinghua, China, in 1994 and 1997, respectively, and the Ph.D. degree from the Department of Electrical Engineering and Computer Sciences, University of California, Berkeley, in 2002.

Since 2002, he has been working as an Assistant Professor in the Department of Electrical Engineering and Computer Sciences and the Laboratory of Information and Decision Systems, Massachusetts Institute of Technology (MIT), Cambridge. His research interests includes information theory, wireless communications, and wireless networks.

Dr. Zheng received the Eli Jury Award from the University of California in 2002, and the IEEE Information Theory Society Paper Award in 2003. 